\title{
High-throughput development of simple sequence repeat markers for genetic diversity research in Crambe abyssinica
}

\author{
Weicong $\mathrm{Qi}^{1 \dagger}$, Feng Lin ${ }^{1 \dagger}$, Yuhe Liu², Bangquan Huang ${ }^{3}$, Jihua Cheng ${ }^{3}$, Wei Zhang ${ }^{4}$ and Han Zhao ${ }^{*}$
}

\begin{abstract}
Background: The allohexaploid Crambe abyssinica (crambe) is an oilseed crop that has been recognized for its potential value in the chemical industry, particularly in terms of producing high-erucic acid content vegetable oil. However, as an understudied crop, improvement of crambe has been hampered by the lack of genetic and genomic information to enhance its yield, oil quality and resistance against biotic and abiotic stress. Development of molecular markers is therefore of great significance to facilitate genetic improvement of crambe.

Results: In this study, high-throughput sequencing was performed to generate sequences for the transcriptome and genome of a widely planted crambe cultivar, Galactica. A total of 186,778 expressed sequence tag (EST) contigs as 8,130,350 genomic contigs were assembled as well. Altogether, 82,523 pairs of primers were designed in the flanking sequences of the simple sequence repeat (SSR) within these contigs. Virtual PCR analysis showed that a fraction of these primers could be mapped onto the genomes of related species of Brassica, including Brassica rapa, B. oleraceae and B. napus. Genetic diversity analysis using a subset of 166 markers on 30 independent C. abyssinica accessions exhibited that 1) $95 \%$ of the designed SSRs were polymorphic among these accessions; 2) the polymorphism information content (PIC) value of the markers ranged from 0.13 to $0.89 ; 3$ ) the genetic distances (coefficient NE172) between accessions varied from 0.06 to 0.36 . Cluster analysis subsequent on the accessions demonstrated consistency with crambe breeding history. F-statistics analysis revealed a moderate level of genetic differentiation in C. abyssinica (Gst $=0.3934)$ and a accordingly low estimated gene flow $(\mathrm{Nm}=0.7709)$.

Conclusion: Application of high-throughput sequencing technology has facilitated SSR marker development, which was successfully employed in evaluating genetic diversity of C. abyssinica as demonstrated in our study. Results showed these molecular markers were robust and provided powerful tools for assessing genetic diversity and estimating crambe breeding history. Moreover, the SSR primers and sequence information developed in the study are freely available to the research community.
\end{abstract}

Keywords: Crambe abyssinica, EST-SSR, SSR, Molecular breeding, Genetic diversity, Next-generation sequencing, de novo assembly

\section{Background}

Crambe abyssinica (crambe) is an allohexaploid $(2 \mathrm{n}=$ $6 x=90)$ with an estimated genome size of approximately $3.5 \mathrm{~Gb}$ based on its $2 \mathrm{C}$-value $(=7.04 \mathrm{pg})[1-5]$. A member of the genus Crambe abyssinica distribute unevenly among four major geographical regions:

\footnotetext{
* Correspondence: zhaohan@jaas.ac.cn

${ }^{\dagger}$ Equal contributors

'Institute of Biotechnology, Provincial Key Laboratory of Agrobiology, Jiangsu

Academy of Agricultural Sciences, Nanjing 210014, China

Full list of author information is available at the end of the article
}

Macronesian, Mediterranean, East Africa, and EuroSiberian-southwest Asia [6]. C. abyssinica originated from the Mediterranean region and has been cultivated mainly for producing high-erucic acid plant oil, a natural product of interest to the chemical industry $[2,3]$. Its breeding and cultivation first started in Europe from the 1900s, and then subsequently spread throughout the world [7]. Previous efforts in improving crambe's agronomic traits included traditional breeding [7], mutagenesis, and transgenesis [7-11]. Despite obvious improvement on agronomic traits, genetic and genomic information on this 
oilseed crop is still largely limited. So far, only a few hundred nucleotide sequences of DNA and RNA in $C$. abyssinica were deposited in public database (National Center for Biotechnology Information), markedly incomparable to other oil crops, e.g. Brassica napus, soy bean, peanut or sunflower. The paucity of available information on nucleotide sequences has hindered its genetic studies, such as molecular marker development, linkage map construction and gene discovery.

The next-generation sequencing (NGS) technologies have significantly increased the speed and throughput of sequence information acquisition, and greatly accelerated the discovery process for molecular markers $e$. $g$. single nucleotide polymorphisms (SNP) and SSR [12, 13]. Although SNP markers are popular and widely applied in major crops, SNP detection usually requires expensive chemistries and equipment which limited its application. SSR markers are technologically less demanding and have advantages including high level of polymorphism, low cost, reproducibility and transferability across species. For example, a total of 82 barley EST-derived SSR primer pairs were tested for transferability to $\mathrm{H}$. chilense, all of which amplified products of correct size from this species [12]. Moreover, in some species, SSRs were found to be more informative than SNPs, for instance Singh et al. [13] compared the use of SSR and SNP markers in estimation of genetic diversity and population structure of Indian rice varieties, and concluded that SSR were more efficient for diversity analysis.

In the study, we have employed the NGS sequencing technology to sequence genomic DNA and EST of Crambe abyssinica with the goals of characterizing simple satellite repeat loci and developing corresponding markers. Our study demonstrated the procedures for developing SSR markers for C. abyssinica, facilitated by the use of the next-generation sequence technology. The developed SSR markers were utilized to evaluate the genetic diversity of crambe accessions, which exhibit a moderate level of genetic differentiation in $C$. abyssinica and a correspondingly estimated gene flow. The nucleotide sequences generated in present study including the SSR-primers and the assembled genomic and transcriptomic contigs are freely available to the research community, and will serve as useful genetic resource for this species.

\section{Results}

De novo assembly of transcriptomic and genomic DNA of Crambe abyssinica

The total RNA of developing crambe seeds (21 days after pollination) was isolated and synthesized cDNA was sequenced by Illumina Pair-End $100 \times 2$, from which a total of $4.0 \mathrm{~Gb}$ sequence data were generated. The deduced coverage of the transcriptome was about $20 \times$. The raw data were processed using Q20 quality control and L40 length filtering. The remaining raw reads were assembled using the Trinity program to generate contigs. A total of 234,622 contigs were generated from the de novo assembly of cDNA. Then, the contigs were filtered using BLASTN (1E-50) to remove those with high similarity. Finally 186,778 contigs $(209 \mathrm{Mb})$ remained for EST-SSR calling. The lengths of the contigs varied from $100 \mathrm{bp}$ to $>10 \mathrm{~kb}$ with the average $1,138 \mathrm{bp}(\mathrm{N} 50=$ $1,428 \mathrm{bp}$ ). The genomic DNA isolated from fresh leaves was sequenced by Illumina similar to that of the cDNA. A total of $33.5 \mathrm{~Gb}$ raw data was obtained, indicating a $9.5 \times$ depth of coverage. The raw data were processed with EST data, except for being assembled by a 'Short Oligonucleotide Analysis Package program de novo' (SOAPdenovo). Finally, 8,130,350 contigs (1.41 Gb) were assembled for genome-SSR calling (Table 1). The average size of the contigs was $186 \mathrm{bp}$, and N50 is $275 \mathrm{bp}$ with the longest sequence length of $8,770 \mathrm{bp}$. Figure 1 showed the distribution of the assembled EST and genomic contigs according to their lengths. The EST contigs between 1 to $2.5 \mathrm{~kb}$ were the most abundant. Among the genomic contigs, those within 0.1 to $0.25 \mathrm{~kb}$ were the most prevail in terms of contig appearance frequency, but the contigs within 0.25 to $0.5 \mathrm{~kb}$ were the most dominant in terms of the ratio in total length.

SSR development, primer design and preliminary selection SSR loci were called using the MISA program from the assembled transcriptomic and genomic contigs. The parameters were designed for identifying perfect di-, tri-, tetra-, penta-, and hexanucleotide motifs with a minimum of $8,5,4,4$, and 3 repeats, respectively. As showed in Table 1, among the de novo assembled contigs, 19,674 cDNA contigs out of 186,778 (10.5\%), and 89,983 genomic contigs out of 8,130,350 (1.1\%) contained at least one SSR locus. On average, there was one SSR locus in every $11.1 \mathrm{~kb}$ cDNA and $16.8 \mathrm{~kb}$ genomic DNA, respectively. Eventually, a total of 22,734 EST derived SSR loci and 97,170 genomic SSRs loci were detected. A total of 274 EST-SSR motifs and 455 genomic SSR motifs were identified. Of the EST-SSR motifs, there were 3 di-, 10 tri-, 24 tetra-, 38 penta and 199 hexa-nuleotide; and of the genomic SSR motifs, there were 3 di-, 10 tri-, 31 tetra-, 89 penta and 322 hexa-nuleotide. The Fig. 2 demonstrated the statistics in the SSR loci detected. Of the EST-SSR loci, trinucleotides accounted for $60 \%$ and thus ranked the most abundant, followed by dinucleotides (21\%), hexanucleotides (11\%), tetranucleotides (5\%), and pentanucleotides (2\%) (Fig. 2). While for the detected genomic-SSR loci, $50 \%$ were dinucleotide motifs, followed by $26 \%$ trinucleotides, $14 \%$ hexanucleotides, $7 \%$ tetranucleotides and $3 \%$ pentanucleotide (Fig. 2). The top ten most abundant nucleotide repeat types in the 
Table 1 Summary of the de novo assembly of transcriptome and genome and SSR loci calling

\begin{tabular}{lll}
\hline & Transcriptome & Genome \\
\hline Raw data & $4.0 \mathrm{~Gb}$ & $33.5 \mathrm{~Gb}$ \\
Total number of contigs & 186,778 & $8,130,350$ \\
Total length of contigs & $209 \mathrm{Mb}$ & $1.41 \mathrm{~Gb}$ \\
Total number of the contigs with & 19,674 & 89,983 \\
SSR locus & & \\
The frequency of SSR occurrence & One locus per & One locus per \\
& $11.1 \mathrm{~kb}$ & $16.8 \mathrm{~kb}$ \\
Contigs N50 & $1,428 \mathrm{bp}$ & $275 \mathrm{bp}$ \\
Average contig length & $1,138 \mathrm{bp}$ & $186 \mathrm{bp}$ \\
Maximum contig length & $16,475 \mathrm{bp}$ & $8,770 \mathrm{bp}$ \\
\hline
\end{tabular}

newly detected SSRs were presented in Fig. 3, with the most common EST-SSR motif being AAG/CTT and the most abundant genomic-SSR motif being AG/CT.

Primer pairs with melting temperatures $\left(\mathrm{T}_{\mathrm{m}}\right)$ within the range of $55{ }^{\circ} \mathrm{C}$ to $61{ }^{\circ} \mathrm{C}$ and with amplicons within a size range of $150 \mathrm{bp}$ to $400 \mathrm{bp}$ were designed from the flanking sequences of the SSR loci by using the Primer 3

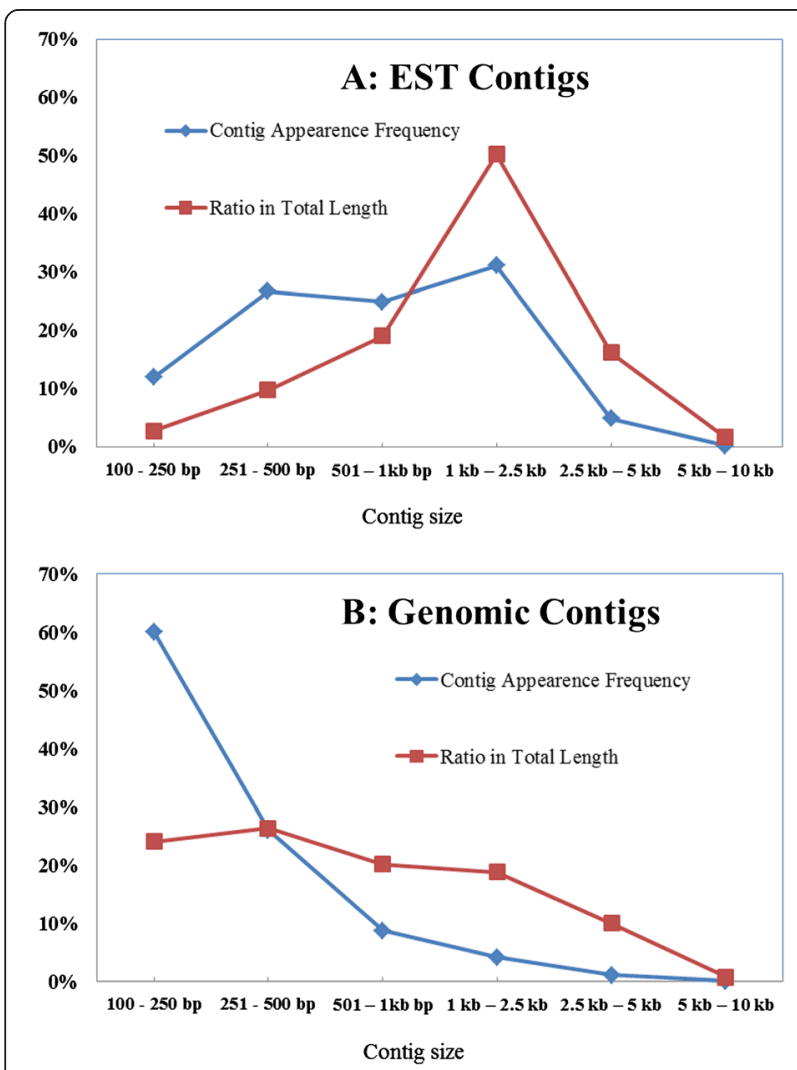

Fig. 1 The distribution of the assembled contigs in different sizes. Chart A and B has showed the size distribution of the assemble EST and genomic contigs respectively. The line in blue indicated the appearance frequencies of the contigs in different size, and the red line showed the corresponding ratio in total length program. Primers were then validated by in silico PCR against the crambe genome contigs per se. Those primer sets amplifying multiple target regions were discarded to ensure the specific locations of the primers. Finally, a total of 3,803 pairs of EST-SSR primers (Additional file 1) and 78,720 genomic-SSR primer pairs (Additional file 2) were obtained and the ratio between EST- and genomic-SSR primer pairs was around 1:20.

\section{Validating the primers with the whole-genome data of Brassicaeace crops}

Brassica rapa, Brassica olecera, and Brassica napus belong to the same family Brassicaceace as crambe. To assess the intergeneric homology of the newly developed crambe SSRs across Brassicaceace, the EST- and genomicSSR primers were tested by in silico PCR against the genome of $B$. rapa, B. oelcera and B. napus. The maximum number of mismatches at the $5^{\prime}$ end is three base pairs, with none allowed at the 3 ' end. The primer pairs that amplified a single band between $200 \mathrm{bp}$ and 500 bp were considered as the preferred molecular markers. Finally, 339 pairs of EST-SSR primers and 3,467 pairs of genomicSSR primers were mapped onto $B$. rapa genome (Additional file 3), 305 and 3,114 on B. olarecea (Additional file 4), 174 and 1,888 on B. napus (Additional file 5). The overlapped primers among three genomes were showed in Fig. 4, where 22 EST-SSR primer pairs and 382 genomicSSR primer pairs overlapped across all three genomes, suggesting the corresponding amplified loci are evolutionarily conserved in the family. According to the physical location, these primer pairs distributed at a frequency of one marker per $10 \mathrm{~kb}$ in Brassica rapa and Brassica olecera (Fig. 5).

\section{Evaluating the diversity of the C. abyssinica germplasm collections by using the newly developed SSR markers} A total of 166 primer pairs (Additional file 6) consisting of 13 EST-SSRs and 153 genomic-SSRs were selected and utilized in the PCR analysis of genomic DNA from 30 crambe accessions to validate the utility and reliability of these SSR markers in C. abyssinica germplasm identification (Table 2). In terms of geographic origins, the accessions can be classified into four groups: the Mediterranean group (accessions from Ethiopia, Spain, and Turkey), the Middle European group (accessions from Ukraine, Former Soviet Union, and Romania), the Western European group (accessions from German and Holland), and the USA group. Amplified products were analyzed by polyacrylamide gel electrophoresis.

Results revealed that the tested EST- and genomic-SSR markers were highly polymorphic among the accessions. The polymorphism information content (PIC) value of the primers varied from 0.13 to 0.89 (Additional file 6). There were nine primer pairs showing no polymorphisms among 


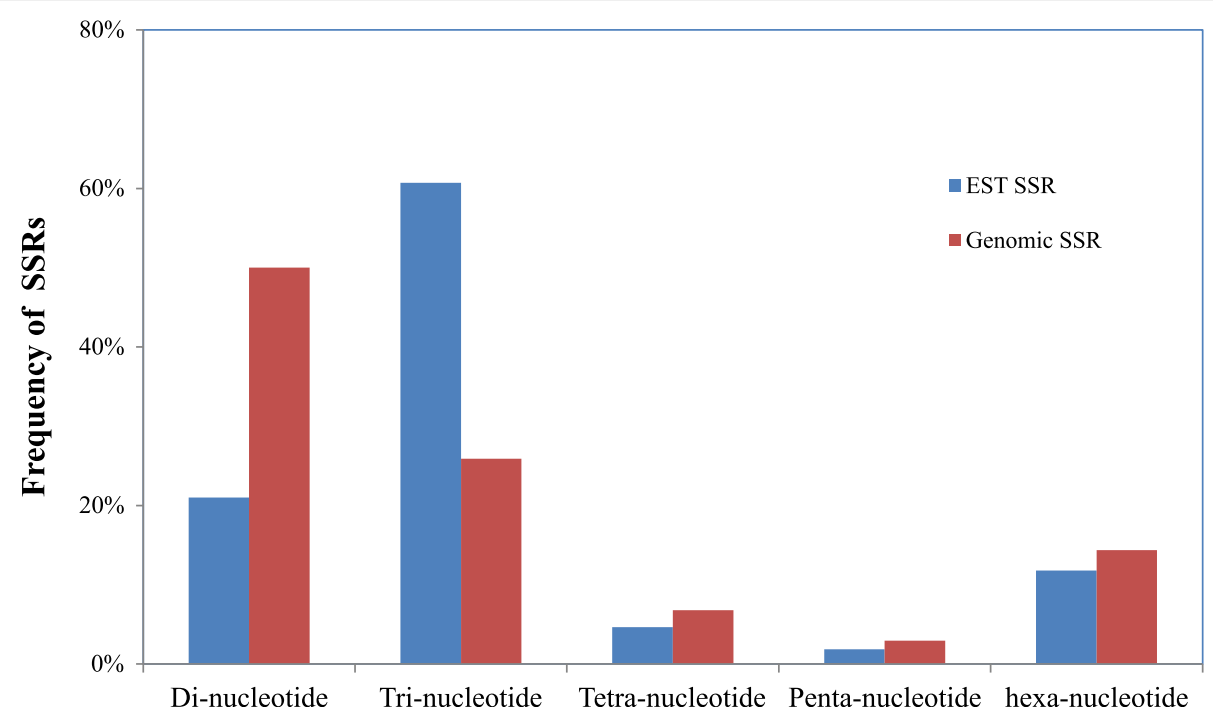

Fig. 2 SSRs Statistics. The frequency distribution of the SSR motif was showed in the chart. The nucleotide repeat motif varied from di-nucleotide to hexa-nucleotide as showed by the $X$ axis. The tri- and di-nucleotide were the most prevail in the EST and genomic SSR respectively

the accessions. As shown in Table 2, a total of 858 alleles were detected in 30 accessions, wherein 77 alleles were determined to be accession-specific and 273 alleles were generally detected in all the accessions.

The genetic distances with the coefficient of NEI72 among accessions ranged from 0.06 to 0.36 (Additional file 7). The largest genetic distance was observed between Galactica and PI306422 from Romania. Unweighted pair group method analysis (UPGMA) was used to cluster the accessions, which indicated that the 30 accessions could be grouped accordingly into two clusters (Fig. 6). Cluster A included all the accessions except Galactica and could be further subdivided into two sub-clusters. Sub-cluster AI contained two groups: Group 1 consisted of the accessions from the origin of C. abyssinica (Mediterranean region) and surrounding areas, whereas Group 2 comprised one from Iowa, US (PI414156), two from Indiana ('Prophet'/PI514650; 'Meyer'/ PI514649) and US, two accessions ('C-22'/PI533664; C-29/ PI533665) from Maryland, US. Sub-cluster AII included three accessions from Maryland ('BelEnzian'/PI533668; 'BelAnn'/PI533667; 'C-37'/), US and one (PI414156) from New Mexico, US and one (PI633197) from Germany. In comparison with other American accessions, the accessions collected from Iowa and Indiana had a closer relationship with the ones from Mediterranean region and surrounding areas.

F-statistics analysis of the PCR results showed a generally moderate level of genetic differentiation among the accessions (Gst $=0.3934$ ), and a corresponding low estimated gene flow $(\mathrm{Nm}=0.7709)$. The accessions from the Mediterranean region and surrounding areas (Europe, Africa, and Asia) showed a similar degree of genetic differentiation ( $\mathrm{Gst}=0.3866$, corresponding to an estimated gene flow $\mathrm{Nm}=0.7934)$. In addition, the accessions from the USA showed a relatively lower level of genetic differentiation and a higher estimated gene flow (Gst $=0.1868$, corresponding estimated gene flow $\mathrm{Nm}=2.1764)$.

\section{Discussion}

In present study, SSR markers for the hexoploid species Crambe abyssinica from Mediterranean region were developed based on de novo assembled cDNA and genomic DNA from cultivar Galactica released by Wageningen University, the Netherlands. The reliability of these newly developed SSR primers was tested via PCR analysis on a total of 30 different crambe germplasm accessions, including the modern cultivars 'Prophet' (PI514650), 'Meyer' (PI514649), 'BelEnzian' (PI533668), 'BelAnn' (PI533667), 'C-22' (PI PI533664), 'C-29' (PI533665), and 'C-37' (PI533666) from the USA and Galactica from the Netherlands. The UPGMA tree plot in Fig. 6 showed a reasonable and conclusive topology that was consistent with the described breeding history of C. abyssinica [14]. For example, the figure showed 'Prophet' and 'Meyer' had closer relationship with the main accessions from Mediterranean and neighboring areas than 'C22,' 'C29,' 'C37', BelEnzian and BelAnn. It automatically reflected the fact that 'Prophet' and 'Meyer' were obtained from mass selection and crossing in two initial accessions from Sweden and Ethiopia; and 'C22,' 'C29, 'C37', BelEnzian and BelAnn also originated from these two initial accessions but with extra breeding process and longer term of selection [15]. The figure also showed that American accessions were rather isolated from those from European, Asian, and 

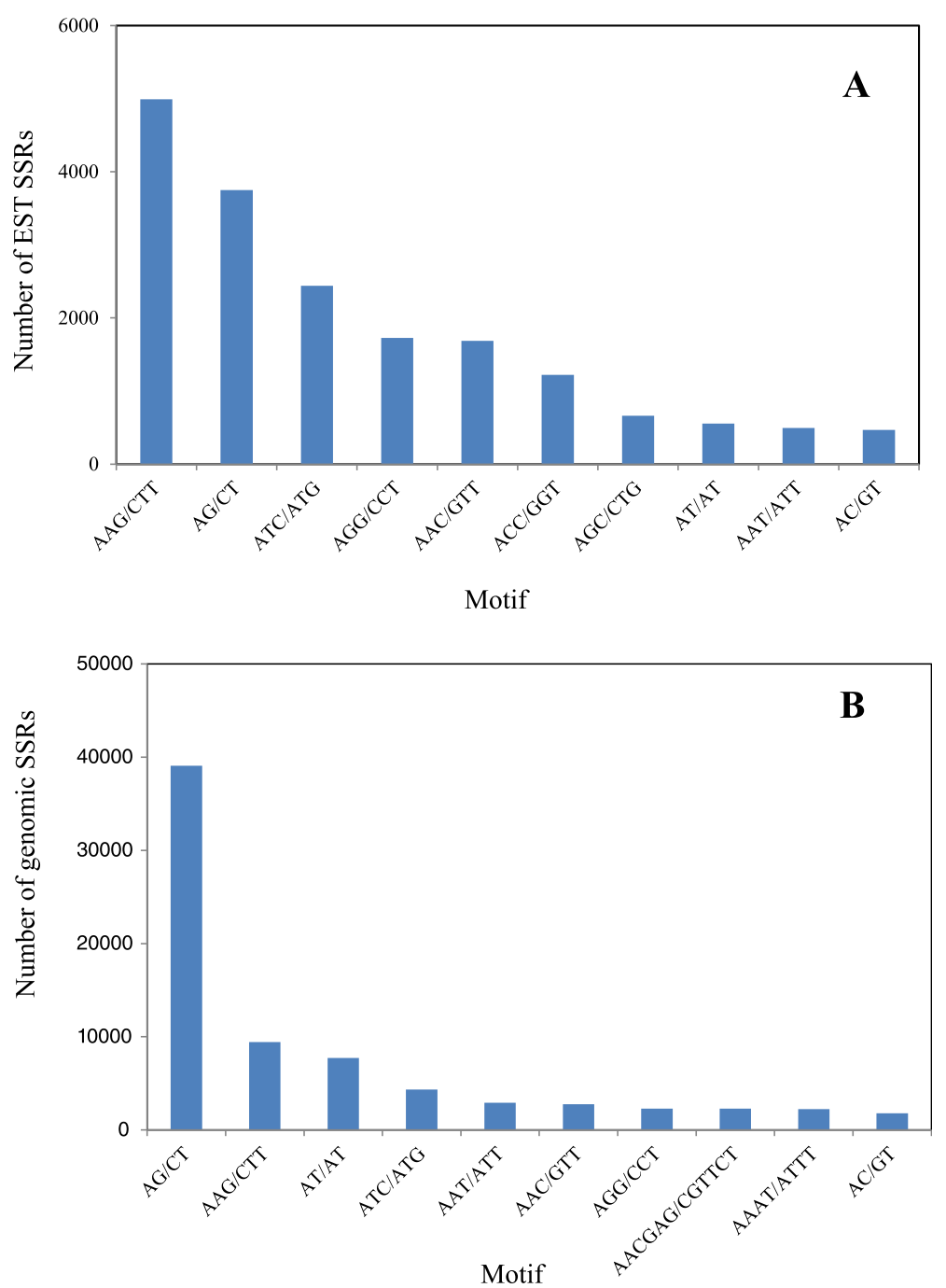

Fig. 3 Distribution of top-10 motifs in EST-SSRs (a) and genomic SSRs (b). The most common EST-SSRs were trinucleotide motif AAG/CTT, whereas the predominant genomic SSRs were dinucleotide motif AG/CT. The X-axis represents the motif sequence, and the Y-axis represents the number of detected SSRs

African accessions. It indicated that although crambe breeding in USA was initiated in the1960s from the germplasms of European origin, the breeding effort since then was rather intensive and relatively independent. German accession PI1633197 was an exception which showed a close relationship with the accession from New Mexico, USA. The information regarding this accession is limited in that it was collected by the Institut fur Pflanzengenetik und Kulturpflanzenforschung, Germany, and later donated to United States Agricultural Department, whereas its place origin was unclear. Based on the estimated genetic distances, we deduced hypothesized that: 1) PI1633197 derived from late-breeding accessions; 2) it originated from a germplasm of the USA. Galactica was also a European cultivar which is known as the latest cultivar. It shared its genetic background with all the accessions from USA, Europe, and Mediterranean region, because it was bred from a European landrace and a late American line [15]. F-statistics analysis based on PCR results indicated a moderate level of genetic differentiation among the 30 accessions examined (Gst = $0.3934)$, and gene flow was low $(\mathrm{Nm}=0.7709)$. Geographical isolation, as well as artificial breeding and selection, might have likely caused the genetic differentiation. The genetic differentiation within the USA accessions (Gst = 0.1868) was quite lower than that of the accessions from the Mediterranean and surrounding areas (Europe, Ethiopia, and Turkey, Gst $=0.3866$ ). These findings indicated that: 1) The cultivation history of C. abyssnica in the USA was relatively shorter than that in the Mediterranean and surrounding area; and 2) The crambe genetic resource in the USA was less abundant. The genetic differentiation 


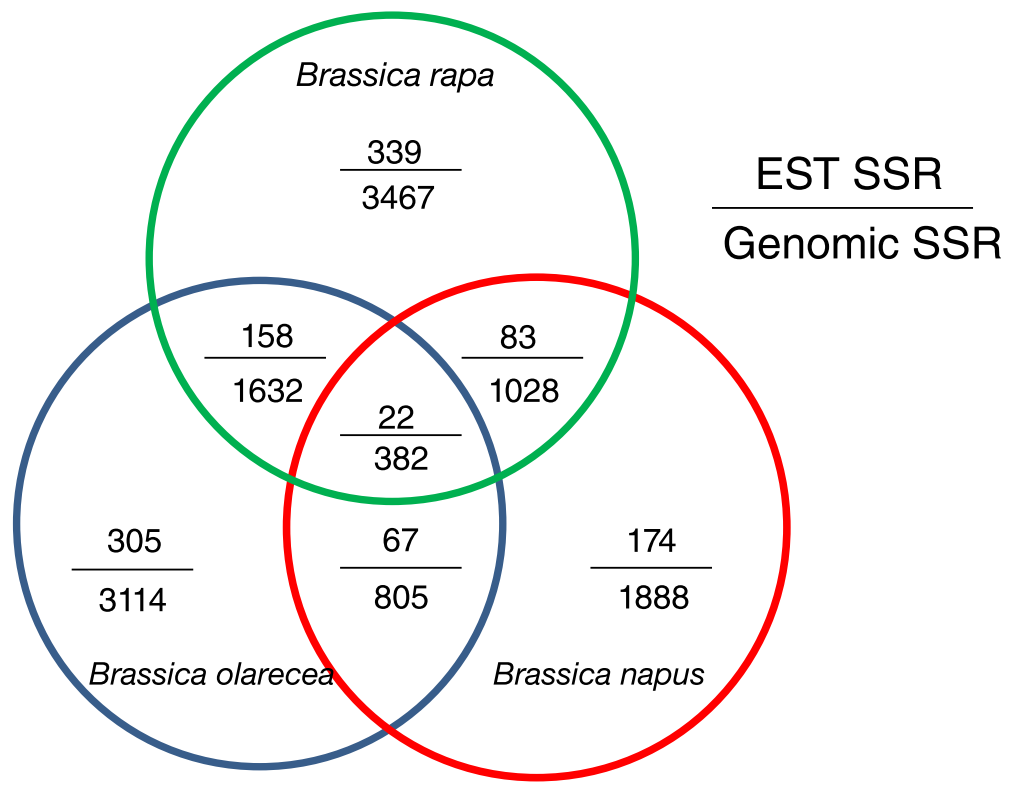

Fig. 4 The number of the primers mapped on genomes of Brassica species. In the chart the numbers of the new primer-pairs which were mapped on B. rapa, B. oleracea and B. napus were showed as well as the number of the overlapped primer-pairs

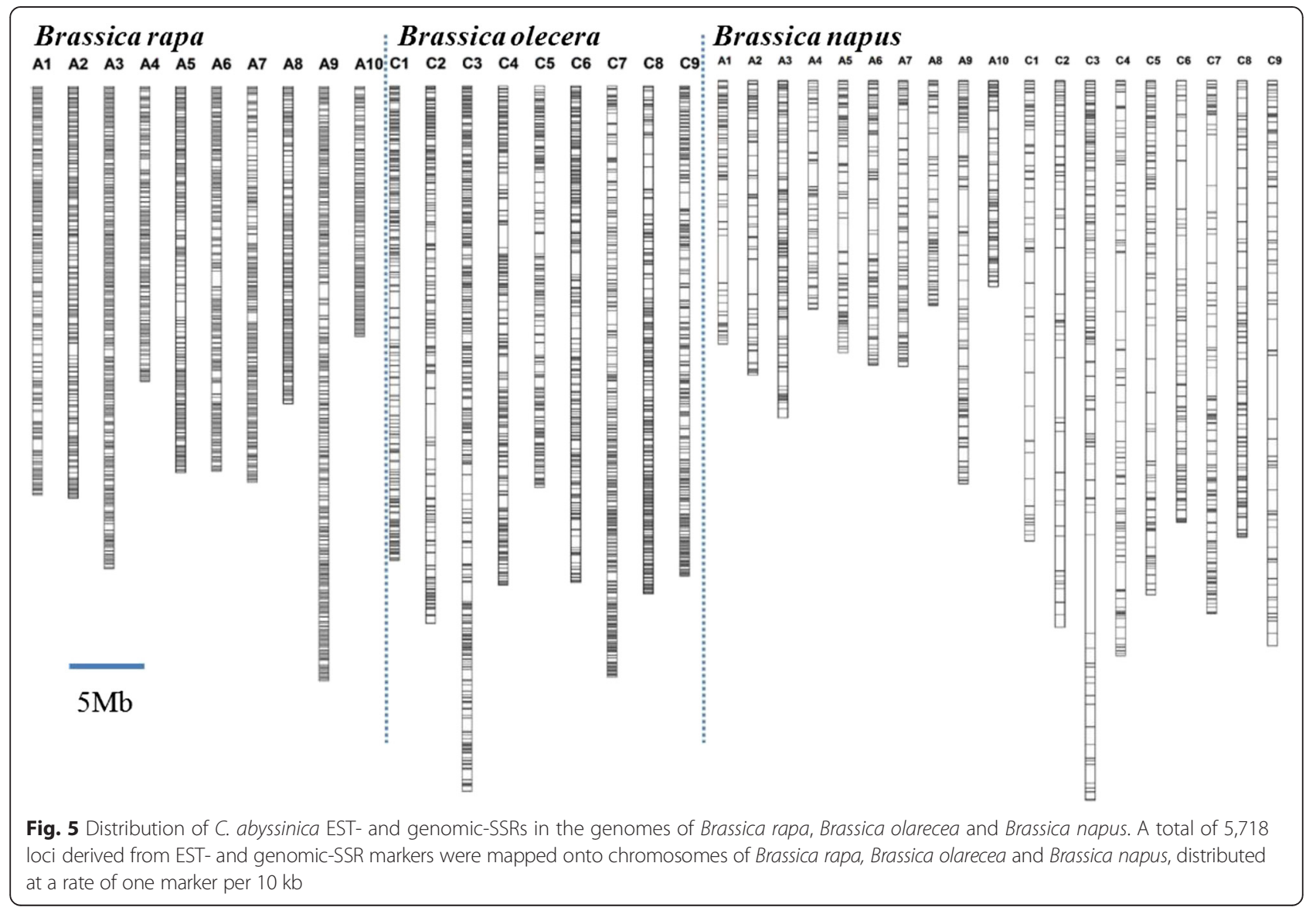


Table 2 Summary of the PCR analysis

\begin{tabular}{|c|c|c|c|c|c|c|c|c|}
\hline PI\# & Origination & Name & Group & TNA & SNA & TTNA & GNA & PGNA \\
\hline 281737 & Ukraine & & Eastern Europe & 569 & 2 & 858 & 273 & 0 \\
\hline 306422 & Romania & & & 609 & 6 & & & 1 \\
\hline 393513 & FSU & & & 609 & 0 & & & 2 \\
\hline 393514 & FSU & & & 613 & 1 & & & 2 \\
\hline 393515 & FSU & & & 603 & 0 & & & 1 \\
\hline 633197 & Germany & CR 1699 & Western Europe & 564 & 2 & & & 3 \\
\hline Galactica & Holland & Galactica & & 464 & 12 & & & 13 \\
\hline 304399 & Denmark & & Northern Europe & 589 & 1 & & & 4 \\
\hline 304400 & Denmark & & & 613 & 5 & & & 1 \\
\hline 360889 & Sweden & & & 627 & 4 & & & 2 \\
\hline 360890 & Sweden & & & 644 & 4 & & & 2 \\
\hline 360891 & Sweden & & & 639 & 1 & & & 1 \\
\hline 360892 & Sweden & & & 651 & 1 & & & 2 \\
\hline 360893 & Sweden & & & 615 & 1 & & & 2 \\
\hline 392071 & Spain & & Mediterranean & 625 & 2 & & & 2 \\
\hline 392072 & Spain & & & 629 & 2 & & & 2 \\
\hline 392326 & Turkey & & & 629 & 6 & & & 4 \\
\hline 392327 & Turkey & & & 629 & 2 & & & 2 \\
\hline 384531 & Ethiopia & Ames 1433 & & 606 & 2 & & & 2 \\
\hline 384532 & Ethiopia & Ames 1434 & & 624 & 4 & & & 2 \\
\hline 384533 & Ethiopia & Ames 1435 & & 623 & 2 & & & 2 \\
\hline 414156 & US, lowa & Ames 1657 & USA & 589 & 1 & & & 1 \\
\hline 514649 & US, Indiana & Meyer & & 562 & 3 & & & 1 \\
\hline 514650 & US, Indiana & Prophet & & 588 & 1 & & & 2 \\
\hline 533664 & US, Maryland & $C-22$ & & 598 & 2 & & & 1 \\
\hline 533665 & US, Maryland & $C-29$ & & 580 & 1 & & & 1 \\
\hline 533666 & US, Maryland & $C-37$ & & 575 & 5 & & & 1 \\
\hline 533667 & US, Maryland & BELANN & & 552 & 1 & & & 4 \\
\hline 533668 & US, Maryland & BELENZIAN & & 587 & 3 & & & 7 \\
\hline 633196 & US, New Mexico & NM 85 & & 557 & 0 & & & 3 \\
\hline
\end{tabular}

Note: The accessions with PI numbers are those from US Department of Agriculture. Abbreviation: TNA tested number of alleles, SNA specific number of alleles, TTNA total number of alleles, GNA general number of alleles, PGNA primers giving not generate alleles

approach based on the polymorphisms in the newly developed SSRs also demonstrated that those markers for $C$. abyssnica and the corresponding primers were reliable and robust.

Among the de novo assembled contigs, it was found that $10.5 \%$ EST-contigs $(19,674$ out of 186,778$)$ contained SSR loci, which was consistent with those of dicotyledonous plants, which ranged from $2.65 \%$ to $16.82 \%$ [16]. The frequency of genomic-SSR occurrence was one per $16.8 \mathrm{~kb}$ which was lower than what has been reported in Brassica species, for instance one locus every $2.5,2.9$ and $2.8 \mathrm{~kb}$ in the genome of B. rapa, $B$. oleracea and $B$. napus respectively [17]. It was mainly because that the mono-nucleotide repeat tandem was excluded in present research. The most abundant dinucleotide motif in crambe genome and EST was poly (AG/CT), which was the same as Arabidopsis genome and ESTs [18], ESTs of Brassica rapa subsp. Pekinensis [19], genome of B. rapa subsp chinensis [20], Medicago truncatula [21] and Raphanus sativus [22]. But in the genomes of $B$. napus, B. rapa subsp. Pekinensis and $B$. oleracea, poly (AT/TA) was the most abundant [17], when poly (AG/CT) ranks as the second [23]. In the EST or genome SSR of Arabidopsis and genus Brassica, the occurrence of poly (GC/CG) was rare, which was as same as what has been found here. Previous studies showed the trinucleotide tandem repeat occurred more frequently in coding region than in non-coding regions 


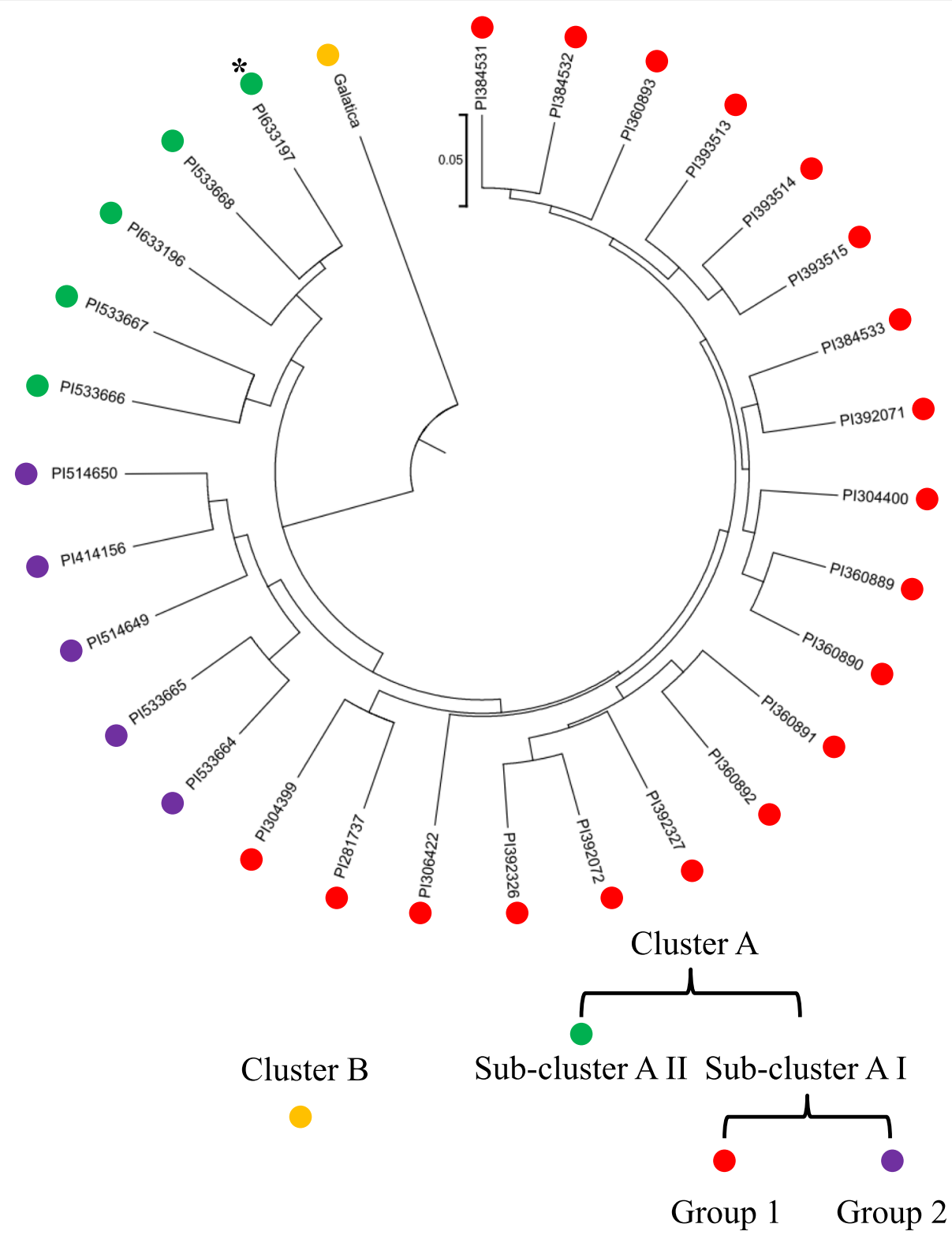

Fig. 6 Tree-plot derived from UPGMA cluster analysis using NEI72 coefficient of SSR markers. The UPGMA (unweighted pair group method analysis) tree-plot was generated using Mega 6.0 cluster analysis based on the genetic distances (calculated with coefficient of NEl72 by NTSYS software) of 30 independent accessions. The names and geographic originations of the accessions were listed in Table 2. The asterisk marks accession Pl633197 from Germany

[24]. And in present research we also found that, relatively, there was more tri-nucleotide SSR in ESTs than in the genomic contigs. The most common triplet repeat detected in crambe EST and genome was poly (AAG/ TTC), which was the same in Arabidopsis [18], B. rapa [19], B. napus, B. olarecea and many other plant species [24]. On the other hand, in C. abyssinica, the SSRs of tetra- and penta-nucleotide motifs were relatively rare when the hexa-nucleotide SSRs were abundant, in comparison with Brassica crops [17]. But as same as Brassica crops, the $\mathrm{A} / \mathrm{T}$ rich motifs rich in for instance AAC,
AAG, AAT, AAAC, AAAG, AAAT, AAAAC, AAAAG, AAAAT and so on were dominant [17]. The PIC values of the crambe SSR varied from 0.13 to 0.89 , which was comparable to those of Brassicaceae species and other plant reported $[20,25]$. Previous research reported the intergeneric transferability of plant SSRs, for example the SSR primers developed from Arabidopsis could be engaged in Brassica species (B. napus, B. rapa, B. oleracea, $B$. nigera, $B$. juncea, and $B$. carinata) and showed polymorphism [26]. In present research, the BLAST analysis also showed that there were a number of crambe SSRs 
could be mapped on the genomes of $B$. rapa, B. oleracea and $B$. napus. This suggested 1 ) the potential intergeneric transferability of the newly developed molecular markers in Brassicaceae family; 2) the whole-genome sequence of Brassica crops [27] or Arabidopsis [28] could serve as references for genomic and genetic research studies on other Brassicaceae species.

There were 166 pairs of primer selected for PCR validation where primers amplified single band in virtual PCR and evenly distributed throughout the Brassica rapa genome. When they were employed for the PCR validation, most of these SSR primers however generated multiple bands. In 30 independent accessions, a total of 858 alleles were detected, and an average of 5 alleles was tested using each primer pair. A total of 273 alleles were generally observed among all accessions, suggesting that these genomic sequences were conserved and duplicated in the crambe genome. Correspondingly, 77 alleles were found accession-specific. Compared to the other accessions, Galactica has the highest number of specific alleles, this finding was reasonable because the markers were designed based on its nucleotide sequences. Also as the latest cultivars, Galactica underwent a longer term of selection, the specific alleles probably correspond artificial selection effect. On the other hand, some primer pairs failed to generate any band patterns (as showed in Table 2) in certain accessions, but no primer pair was unable to generate any polymorphic bands across accessions. This observation may be attributable to various technical reasons such as the primers were designed to cover splice sites; a large intron was present, or the cultivars harbored presence/absence variation. In the future, more cultivars will be sequenced with large genetic distance to better elucidate SSR locus variation and effectively developed corresponding SSR markers.

\section{Conclusion}

The present study has developed a large set of SSR markers for C. abyssinica using high-throughput transcriptome and genome sequencing technologies. 166 of the identified primer pairs were used in the PCR analysis on 30 different accessions. Results showed that: 1) $90 \%$ of the primers generated polymorphic bands; 2) the PIC value of the primers ranged from 0.13 to 0.89 ; and 3) the genetic distances between accessions ranged from 0.06 to 0.36 . Cluster analysis based on genetic distances demonstrated that the accessions could be classified into a manner that was consistent with crambe breeding history. F-statistics analysis of the PCR results showed that the genetic differentiation of C. abyssinica (Gst) was 0.3934, and its corresponding estimated gene flow (Nm) was 0.7709 . These results suggested that due to geographical isolation and artificial selection, $C$. abyssinica has adapted moderate level of genetic differentiation and gene flow. SSR primers and sequence information developed in the present study are freely available to the research community, serving as a useful and robust resource for molecular taxonomy studies, linkage map construction, and molecular marker-assisted breeding.

\section{Methods}

\section{Plant materials and isolation of DNA and RNA}

C. abyssinica cultivar Galactica was used for genome and cDNA sequencing. Another 29 accessions (acquired from USDA germplasm resources) were used for SSR testing and cluster analysis. Crambe seeds were germinated in Petri dishes with two layers of fully wetted filter paper and kept at $25{ }^{\circ} \mathrm{C}$ in the dark. Upon radical emergence, the seedlings were transferred to soil and kept in a greenhouse with an average temperature of $20^{\circ} \mathrm{C}$.

Genomic DNA was isolated from young leaves of 30day-old crambe plants after seed germination following the method described by Aldrich and Cullis (1993), but with $1 \%(\mathrm{w} / \mathrm{v})$ polyvinylpyrrolidone-10 in a DNA extraction buffer.

Total RNA of developing seeds was extracted from bulked seeds of T0 plants [(10 seeds per plant, 21 days after flowering (DAF)] using RNeasy Plant Mini Kits (Qiagen, Germany), following the manufacturer's instructions. The isolated RNA was treated with RNase-free TURBO DNase (Ambion, USA) to remove residual genomic DNA. First-strand cDNA synthesis was conducted using $20-\mu \mathrm{L}$ reaction mixtures containing $1 \mu \mathrm{g}$ of total RNA with iScript ${ }^{\text {twi }} c$ DNA Synthesis Kit (Bio-rad, USA).

\section{Illumina sequencing}

Illumina sequencing was conducted at Berry Genomic Ltd., in Beijing, China, following the manufacturer's instructions (Illumina, San Diego, CA). mRNA with a poly (A) tail was isolated from $20 \mu \mathrm{g}$ of total RNA using Seramag magnetic oligo (dT) beads (Illumina). To avoid priming bias, the purified mRNA was first fragmented into small pieces (100-400 bp) using divalent cations at $94{ }^{\circ} \mathrm{C}$ for $5 \mathrm{~min}$. Using random hexamer primers (Illumina), the double-stranded cDNA was synthesized using the SuperScript double-stranded cDNA synthesis kit (Invitrogen, CA). The synthesized cDNA was subjected to end-repair and phosphorylation, and then the repaired cDNA fragments were 3 ' adenylated by using Klenow Exo- (3' to 5' exo minus, Illumina). Illumina paired-end adapters were ligated to the ends of these 3 '-adenylated cDNA fragments. To select the proper templates for downstream enrichment, the products of the ligation reaction were purified on $2 \%$ agarose gel. The cDNA fragments (about $200 \mathrm{bp}$ in size) were excised from the gel. Fifteen rounds of PCR amplification were conducted to enrich the purified cDNA template using PCR primers PE 1.0 and 2.0 (Illumina) using Phusion ${ }^{\circ}$ DNA polymerase. Finally, the 
cDNA library was constructed using 200-bp insertion fragments. After validating on an Agilent Technologies 2100 Bio-analyzer, the library was sequenced on an Illumina HiSeq TM 2000 system (Illumina Inc., San Diego, CA, USA) using the following workflow: template hybridization, isothermal amplification, linearization, blocking, sequencing primer hybridization, and sequencing on the sequencer for read 1. After completion of the first read, the template scan was regenerated in situ to enable a second read from the opposite end of the fragments. Once the original templates were cleaved and removed, the reverse strands were subjected to sequencing-by-synthesis. Genomic DNA was also fragmented into $100-400$ bp segments and subjected to the same library construction and sequencing.

\section{De novo assembly of cDNA}

A next-generation variant calling tool was used for SSR discovery [29]. Prior to the assembly, we conducted a stringent filtering process of raw sequencing reads. The reads with $>10 \%$ of bases with a quality score of $\mathrm{Q}<20$, non-coding RNA (such as rRNA, tRNA, and miRNA) ambiguous sequences represented as "N" and adaptor contamination were removed. De novo assembly of transcriptome and genome was performed with Trinity and SOAPdenovo2, respectively, using the de Bruijn graph method and default settings except for the K-mer value. The k-mer value with the best N50 size was selected for final assembly.

\section{Development and detection of SSR markers}

The MISA (http://pgrc.ipk-gatersleben.de/misa/) script was used to identify microsatellites in the unigenes and assembled genome contigs. The software was used to design PCR primers. Forward and reverse SSR primer pairs based on the flanking sequences of the SSR loci were designed by running the software in batch mode. The primers varied in length from 18 to $20 \mathrm{bp}$ (the optimal length: $20 \mathrm{bp}$ ), with $\mathrm{GC}$ contents varying between $45 \%$ and $65 \%$ (optimal GC content: $50 \%$ ). These preliminary primer pair sequences were validated by in silico PCR analysis against the B. rapa genome and all possible amplifications were determined using the BLASTN program. Genome sequences of these three species were download from the public database (Brassica rapa: http://www.ncbi.nlm.nih.gov/genome/ 229; Brassica oleracea: http://www.ncbi.nlm.nih.gov/ genome/10901; Brassica napus: http://www.ncbi.nlm. nih.gov/genome/?term=brassica\%20napus).

PCR amplification was conducted in the following conditions: DNA was denatured at $94{ }^{\circ} \mathrm{C}$ for $4 \mathrm{~min}$; followed by $35-40$ cycles of $94{ }^{\circ} \mathrm{C}$ for $30 \mathrm{~s}, 55^{\circ} \mathrm{C}-60{ }^{\circ} \mathrm{C}$ for $30 \mathrm{~s}$, and $72{ }^{\circ} \mathrm{C}$ for $2 \mathrm{~min}$; and a final extension at $72{ }^{\circ} \mathrm{C}$ for $10 \mathrm{~min}$. The PCR products were analyzed by electrophoresis on $8.0 \%$ non-denaturing polyacrylamide gels with ethidium bromide. The band sizes were determined against a DNA ladder. A total of 19 ESTSSR primers pair and 213 genomic SSR primer-pairs were used in the experiment. The amounts of each primer pair were based on the ratio between the detected EST-SSRs and genomic SSRs.

\section{Data analysis}

The PIC value was calculated using the formula (PIC $\left.=1-\sum(\mathrm{Pi})^{2}\right)$, where $\mathrm{Pi}$ is the proportion of samples carrying the allele of a particular locus. The genetic distances were calculated with the NTSYSpc (version $2.1 \mathrm{~s}$ ) software (http://www.exetersoftware.com/cat/ntsyspc/ntsys pc.html http://www.exetersoftware.com/cat/ntsyspc/ntsysp c.html) with the coefficient of NEI72. In addition, UPGMA was adopted for cluster analysis and to generate a representative tree plot. By assuming Hardy-Weinberg disequilibrium, the POPGENE software (version 1.31; https:// www.ualberta.ca/ fyeh/popgene_download.html) was used to calculate the Gst and $\mathrm{Nm}$ using the formula: $\mathrm{Nm}=0.5$ $(1$ - Gst)/Gst.

\section{Additional files}

Additional file 1: The total dataset of the EST-SSR primer sequence.
$(X L S X 172 \mathrm{~kb})$

Additional file 2: The total dataset of the genomic-SSR primer sequence. (XLSX $3479 \mathrm{~kb}$ )

Additional file 3: Primers mapped on Brassica rapa genome (XLSX $177 \mathrm{~kb}$ ) Additional file 4: Primers mapped on Brassica oleracea genome (XLSX $174 \mathrm{~kb}$ )

Additional file 5: Primer mapped on Brassica napus genome (XLSX $101 \mathrm{~kb}$ ) Additional file 6: The 166 primer-pairs validated by PCR. (XLSX 25 kb) Additional file 7: The genetic distances with the coefficient of NEI72 among accessions. (XLSX $14 \mathrm{~kb}$ )

\section{Abbreviations}

EST, expressed sequence tag; NGS, next-generation sequencing; PIC, polymorphism information content; RAPD, random amplified polymorphic DNA; SNP, single nucleotide polymorphisms; SSR, simple sequence repeat; UPGMA, unweighted pair group method analysis

\section{Acknowledgements}

The experiment material crambe accessions 'Galactica' and the others were kindly provided by Wageningen University and United States Department of Agriculture, respectively.

\section{Funding}

This work was supported by Grant from Natural Science Foundation of Jiangsu Province, China (BK20141385), Jiangsu Agriculture Science and Technology Innovation Fund [cx (14)2009].

Availability of data and materials

The primers designed in this article are included within the article and its additional files.

\section{Authors' contributions}

WQ conceived the study and participated in its design, practical work, data analysis, and manuscript writing. YL participated in data analysis and the 
most of the bioinformatics work. FL, YL, BH, WZ, and JC participated in the discussion and provided intellectual input to this research. $\mathrm{HZ}$ conceived the study, participated in its design and coordination, and manuscript writing. All the authors have read and approved the final manuscript.

\section{Competing interests}

The authors declare that they have no competing interests.

\section{Consent for publication}

Not applicable.

\section{Ethics approval and consent to participate}

Not applicable.

\section{Author details}

'Institute of Biotechnology, Provincial Key Laboratory of Agrobiology, Jiangsu Academy of Agricultural Sciences, Nanjing 210014, China. ${ }^{2}$ Department of Crop Sciences, University of Illinois, Urbana-Champaign, IL 61801, USA. ${ }^{3}$ College of Life Science, Hubei University, Wuhan 430062, China. ${ }^{4}$ Waksman Institute of Microbiology, Rutgers University, 190 Frelinghuysen Road, Piscataway, NJ 08854, USA.

Received: 6 April 2016 Accepted: 8 June 2016

Published online: 18 June 2016

\section{References}

1. Rudloff E, Wang Y. Crambe. In: Kole C, editor. Wild Crop Relatives: Genomic and Breeding Resources. Berlin: Springer; 2011. p. 97-116.

2. White GA, Higgins JJ. Culture of Crambe: A new industrial oilseed crop. Washington: Agricultural Research Service, U.S. Dept. of Agriculture; 1966.

3. Bruun J, Matchett J. Utilization potential of Crambe abyssinica. J Am Oil Chem Soc. 1963:40(1):1-5.

4. Falasca SL, Flores N, Lamas MC, Carballo SM, Anschau A. Crambe abyssinica: An almost unknown crop with a promissory future to produce biodiesel in Argentina. Int J Hydrogen Energy. 2010;35(11):5808-12.

5. Marie D, Brown SC. A cytometric exercise in plant DNA histograms, with 2C values for 70 species. Biol Cell. 1993;78(1-2):41-51.

6. Francisco-Ortega J, Fuertes-Aguilar J, Gómez-Campo C, Santos-Guerra A Jansen RK. Internal Transcribed Spacer Sequence Phylogeny ofCrambeL. (Brassicaceae): Molecular Data Reveal Two Old World Disjunctions. Mol Phylogenet Evol. 1999;11(3):361-80.

7. Mastebroek HD, Wallenburg SC, van Soest LJM. Variation for agronomic characteristics in crambe (Crambe abyssinica Hochst. ex Fries). Ind Crop Prod. 1994;2(2):129-36

8. Cheng J, Salentijn EMJ, Huang B, Denneboom C, Qi W, Dechesne AC, Krens FA, Visser RGF, van Loo EN. Detection of induced mutations in CaFAD2 genes by next-generation sequencing leading to the production of improved oil composition in Crambe abyssinica. Plant Biotechnol J. 2015; 13(4):471-81.

9. Li X, van Loo EN, Gruber J, Fan J, Guan R, Frentzen M, Stymne S, Zhu L-H. Development of ultra-high erucic acid oil in the industrial oil crop Crambe abyssinica. Plant Biotechnol J. 2012;10(7):862-70.

10. Vargas-Lopez JM, Wiesenborn D, Tostenson K, Cihacek L. Processing of crambe for oil and isolation of erucic acid. J Amer Oil Chem Soc. 1999; 76(7):801-9.

11. Chhikara S, Dutta I, Paulose B, Jaiwal PK, Dhankher OP. Development of an Agrobacterium-mediated stable transformation method for industrial oilseed crop Crambe abyssinica 'BelAnn'. Industrial Crops and Products. 2012;37(1):457-65

12. Castillo A, Budak H, Varshney RK, Dorado G, Graner A, Hernandez P. Transferability and polymorphism of barley EST-SSR markers used for phylogenetic analysis in Hordeum chilense. BMC Plant Biol. 2008;8:97.

13. Singh N, Choudhury DR, Singh AK, Kumar S, Srinivasan K, Tyagi RK, Singh NK, Singh R. Comparison of SSR and SNP markers in estimation of genetic diversity and population structure of Indian rice varieties. PLoS One. 2013; 8(12):e84136.

14. Stymne S, Dyer J. Oil crop platforms for industrial uses, vol. 5. University of York: CPLPRESS; 2007

15. Carlsson AS. Plant oils as feedstock alternatives to petroleum - A short survey of potential oil crop platforms. Biochimie. 2009;91(6):665-70.
16. Kumpatla SP, Mukhopadhyay S. Mining and survey of simple sequence repeats in expressed sequence tags of dicotyledonous species. Genome. 2005:48(6):985-98.

17. Shi J, Huang S, Zhan J, Yu J, Wang X, Hua W, Liu S, Liu G, Wang H. GenomeWide Microsatellite Characterization and Marker Development in the Sequenced Brassica Crop Species. DNA Res. 2014;21(1):53-68.

18. Cardle L, Ramsay L, Milbourne D, Macaulay M, Marshall D, Waugh R. Computational and Experimental Characterization of Physically Clustered Simple Sequence Repeats in Plants. Genetics. 2000;156(2):847-54.

19. Ding Q, Li J, Wang F, Zhang Y, Li H, Zhang J, Gao J. Characterization and Development of EST-SSRs by Deep Transcriptome Sequencing in Chinese Cabbage (Brassica rapa L. ssp. pekinensis). Int J Genomics. 2015;2015:11.

20. Song X, Ge T, Li Y, Hou X. Genome-wide identification of SSR and SNP markers from the non-heading Chinese cabbage for comparative genomic analyses. BMC Genomics. 2015;16(1):1-18.

21. Gupta S, Prasad M. Development and characterization of genic SSR markers in Medicago truncatula and their transferability in leguminous and nonleguminous species. Genome. 2009;52(9):761-71.

22. Shirasawa K, Oyama M, Hirakawa H, Sato S, Tabata S, Fujioka T, KimizukaTakagi C, Sasamoto S, Watanabe A, Kato M, et al. An EST-SSR Linkage Map of Raphanus sativus and Comparative Genomics of the Brassicaceae. DNA Res. 2011:18(4):221-32

23. Cheng X, Xu J, Xia S, Gu J, Yang Y, Fu J, Qian X, Zhang S, Wu J, Liu K. Development and genetic mapping of microsatellite markers from genome survey sequences in Brassica napus. Theor Appl Genet. 2009;118(6):1121-31.

24. Kalia R, Rai M, Kalia S, Singh R, Dhawan AK. Microsatellite markers: an overview of the recent progress in plants. Euphytica. 2011;177(3):309-34.

25. Varshney RK, Graner A, Sorrells ME. Genic microsatellite markers in plants: features and applications. Trends Biotechnol. 2005;23(1):48-55.

26. Westman AL, Kresovich $\mathrm{S}$. The potential for cross-taxa simple-sequence repeat (SSR) amplification between Arabidopsis thaliana L. and crop brassicas. Theor Appl Genet. 1998;96(2):272-81.

27. Wang $X$, Wang $H$, Wang J, Sun $R$, Wu J, Liu S, Bai $Y$, Mun J-H, Bancroft I, Cheng F,' et al. The genome of the mesopolyploid crop species Brassica rapa. Nat Genet. 2011:43(10):1035-9.

28. Analysis of the genome sequence of the flowering plant Arabidopsis thaliana. Nature 2000, 408(6814):796-815.

29. LV Y, Liu Y, Zhao H. mlnDel: a high-throughput and efficient pipeline for genome-wide InDel marker development. BMC Genomics. 2016;17(1):290.

\section{Submit your next manuscript to BioMed Central and we will help you at every step:}

- We accept pre-submission inquiries

- Our selector tool helps you to find the most relevant journal

- We provide round the clock customer support

- Convenient online submission

- Thorough peer review

- Inclusion in PubMed and all major indexing services

- Maximum visibility for your research

Submit your manuscript at www.biomedcentral.com/submit
) Biomed Central 